\title{
CORRECTION
}

\section{Correction to: Disrupted intraflagellar transport due to IFT74 variants causes Joubert syndrome}

Minna Luo, Zaisheng Lin, Tian Zhu, Minjun Jin, Dan Meng, Ruida He, Zongfu Cao, Yue Shen, Chao Lu, Ruikun Cai, Yong Zhao, Xueyan Wang, Hui Li, Shijing Wu, Xuan Zou, Guanjun Luo, Li Cao, Min Huang, Huike Jiao, Huafang Gao, Ruifang Sui, Chengtian Zhao, $\mathrm{Xu}$ Ma and Muqing Cao (D)

Genetics in Medicine (2021) 23:1175; https://doi.org/10.1038/s41436-021-01191-0

Correction to: Genetics in Medicine 2021; https://doi.org/10.1038/ s41436-021-01106-Z; published online 02 February 2021
Due to a processing error, the online graphical abstract was not given.

The original article has been corrected.
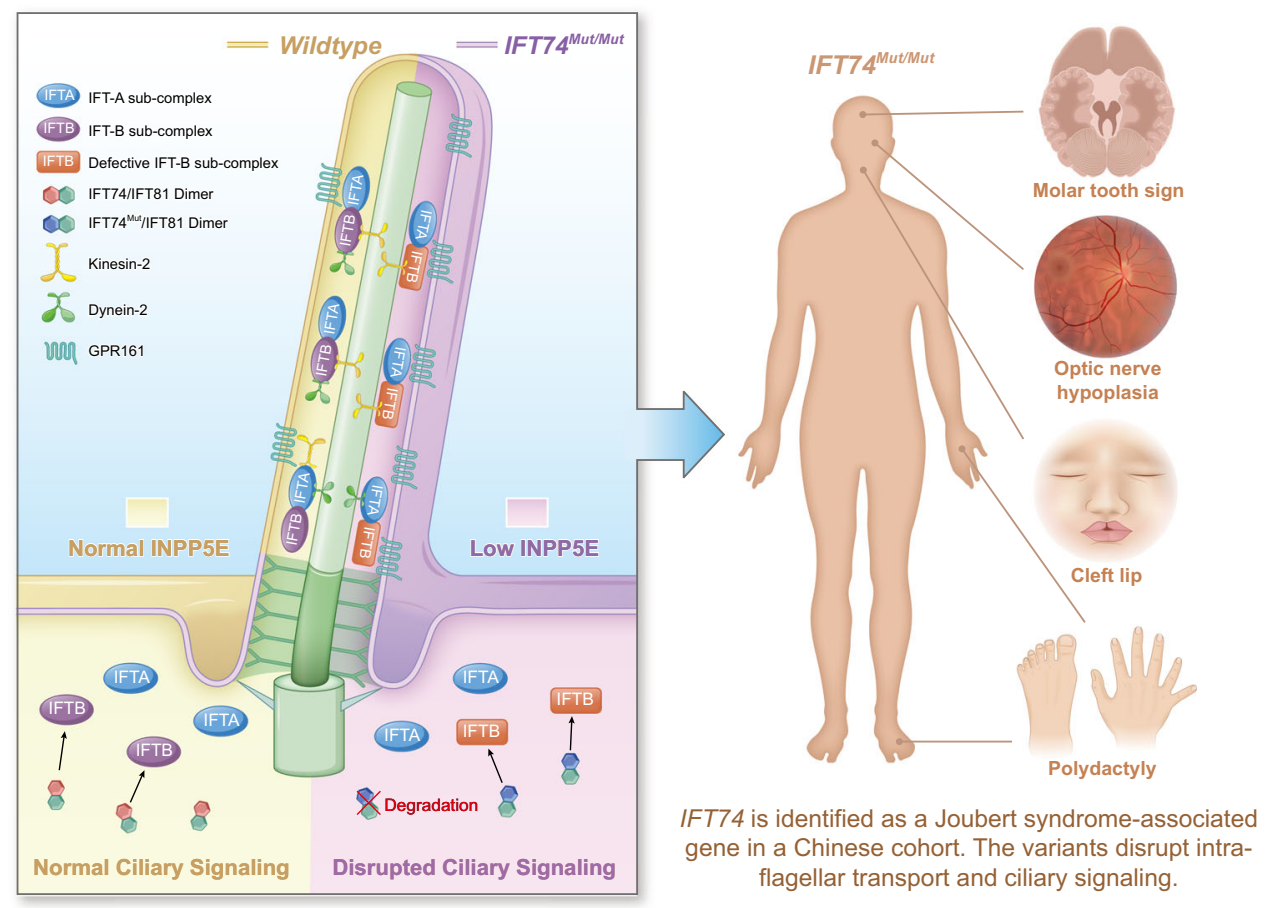

Published online: 10 May 2021 